\title{
Comparative Assessment of Physico-Chemical Parameters of Lotic and Lentic Zone of River Betwa in Jhansi (U.P.), India \\ *Umesh Kumar Mishra and Vijay Kumar Yadav
}

\author{
Department of Zoology \\ Bipin Bihari (P.G.) College, \\ JHANSI (U.P.) INDIA \\ ${ }^{*}$ Corresponding Author \\ E-mail : umeshbetwa@gmail.com
}

Received : 28.09.2020; Revised : 15.10.2020; Accepted : 29.10.2020

\begin{abstract}
Water is the most precious natural gift given by God to human race. Its purity is also important for everyone. For the present study two sampling stations, Baratha village and Parichha dam head were selected for comparative assessment of physico-chemical parameters of lotic and lentic zones on Betwa river. Both these sampling stations are located at Jhansi (UP) India. Samples were collected regularly for one complete year from July 2018 to June 2019. Eighteen water parameters and four hundred thirty two samples were analysed. The overall mean ranges of these parameters at Baratha village and Parichha dam head were notified as electric conductivity $(518.66 \pm 43.47)$ and $(528.75 \pm 59.65), \mathrm{pH}(8.09 \pm 0.36)$ and $(8.14 \pm 0.26)$, water temperature (24.37 \pm 4.14$)$ and $(23.78 \pm 4.12)$, transparency (54.91 \pm 4.97$)$ and (41.61 \pm 3.83$)$, turbidity (30 \pm 12.38$)$ and (35.25 \pm 16.38$)$, TDS (265.33 \pm 16.74$)$ and $(267.66 \pm 25.42)$, TSS $(66.5 \pm 12.52)$ and $(69.83 \pm 14.45)$, TS $(331.83 \pm 28.76)$ and (337.5 \pm 39.04$)$, DO $(6.76 \pm 0.68)$ and $(6.72 \pm 0.86)$, total alkalinity $(141.58 \pm 22.74)$ and $(142.25 \pm 29.37)$, total hardness $(150.08 \pm 22.70)$ and (153.66 \pm 20.55$), \mathrm{Ca}(29.04 \pm 2.32)$ and (29.84 \pm 5.38$), \mathrm{Mg}(13.51 \pm 2.01)$ and (14.32 \pm 2.20$)$, chloride (24.77 \pm 3.29$)$ and (26.85 \pm 3.99$)$, BOD (4.08 \pm 0.71$)$ and $(4.15 \pm 1.16), \mathrm{COD}(11.64 \pm 1.02)$ and $(12.82 \pm 1.20), \mathrm{NO}_{3}(1.64 \pm 0.37)$ and $(1.93 \pm 0.59), \mathrm{PO}_{4}(0.46 \pm 0.21)$ and $(1.33 \pm 0.29)$. All these findings concluded that the lentic zone (Parichha) is more polluted than the lotic zone (Baratha). However all the observed values are in the range of permissible limits, so both zones can be used for irrigation and fisheries sector.

\section{Introduction}

Rivers are the largest source of inland water. Inland water resources have always been known as an essential catalyst that plays a dominant role in irrigation, industrial needs and drinking purpose.

Working area. The Baratha village is situated in Jhansi district along the bank of Betwa river. It is about 15 kilometer away from Jhansi city and GPS coordinates are $25^{\circ} 28^{\prime} 59.7396^{\prime \prime} \mathrm{N}, 78^{\circ} 43^{\prime} 49.2096^{\prime \prime} \mathrm{E}$. It is a gram panchayat village with total population of approximately 3,835 people. The other selected sampling station, Parichha dam head is very well famous landing spot situated about 25 kilometer away from Jhansi city on Jhansi-Kanpur National highway number 25. It is located along the bank of Betwa river with total population of approximately 7,840 people. There is a very popular active thermal power plant which is used for electricity generation. The GPS coordinates of this location are $25^{\circ}$ 28' 45.48' N, 78 42' 39.24" E.

Both the above reported sampling stations are actively used for irrigation, fisheries, drinking, industrial and other domestic purposes. Physico-chemical ranges of any water plays a deciding factor whether water is
Systematic Profile of Sampling Stations.

\begin{tabular}{l|l|l|l}
\hline $\begin{array}{l}\text { Name of } \\
\text { Sampling } \\
\text { Station }\end{array}$ & Stream & Status & $\begin{array}{c}\text { Cityl } \\
\text { District }\end{array}$ \\
\hline $\begin{array}{l}\text { (A)- } \\
\text { Baratha } \\
\text { Village }\end{array}$ & Upstream & Lotic & Jhansi \\
\hline $\begin{array}{l}\text { (B)- } \\
\text { Parichha } \\
\text { dam Head }\end{array}$ & Downstream & Lentic & Jhansi \\
\hline
\end{tabular}

suitable or not for different purposes. Hence testing of water is very important to check the purity and quality of any aquatic zone, whether it is lotic or lentic. In Bundelkhand region, many workers have done their work on the water of Betwa River $5,6,8,10,12,17$.

\section{Material and Methods}

Sample collection and analytical technique

The water samples were collected during morning 


\begin{tabular}{|c|c|c|c|c|c|c|c|c|c|c|c|c|c|c|c|c|c|c|}
\hline 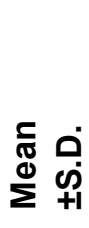 & $\begin{array}{l}\text { f } \\
\dddot{y} \\
+ \\
+ \\
0 \\
\infty \\
\infty \\
i\end{array}$ & $\infty$ & 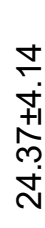 & $\begin{array}{l}\hat{\sigma} \\
+ \\
+ \\
+ \\
\dot{+} \\
\dot{0}\end{array}$ & 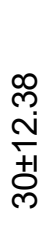 & 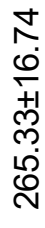 & 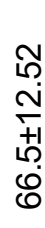 & 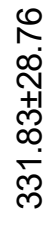 & $\begin{array}{l}\stackrel{8}{0} \\
0 \\
0 \\
0 \\
0 \\
0 \\
0\end{array}$ & $\underset{f}{\square}$ & 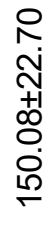 & 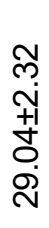 & 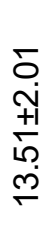 & $\begin{array}{l}\stackrel{9}{ } \\
m \\
+1 \\
\stackrel{N}{N} \\
\dot{N}\end{array}$ & $\begin{array}{l}\nwarrow \\
0 \\
+ \\
+1 \\
0 \\
0 \\
\dot{\sigma}\end{array}$ & 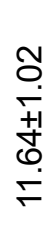 & 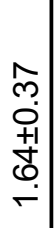 & $\begin{array}{l}\bar{N} \\
0 \\
+1 \\
0 \\
0 \\
0\end{array}$ \\
\hline કํㅇㅇㅇ & 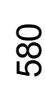 & $\stackrel{+}{+}$ & స్ & స్ & దొ & 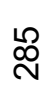 & 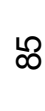 & P & مْ & 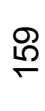 & $\stackrel{?}{\sim}$ & $\frac{\text { q }}{\dot{m}}$ & $\begin{array}{l}\text { مी } \\
\text { مْ }\end{array}$ & $\begin{array}{l}\infty \\
\infty \\
\stackrel{0}{N}\end{array}$ & '. & $\stackrel{\sim}{n}$ & $\stackrel{\bar{N}}{N}$ & 咅 \\
\hline 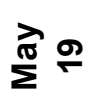 & 周 & $\stackrel{\bullet}{\infty}$. & กิ & $\begin{array}{l}\infty \\
\text { ஸ̂. }\end{array}$ & প্ల & 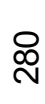 & $\stackrel{\infty}{\sim}$ & 足 & $\stackrel{⿱}{\circ}$ & $\stackrel{\infty}{\infty}$ & $\underset{\varpi}{\varpi}$ & $\begin{array}{l}\text { Lे } \\
\stackrel{m}{m}\end{array}$ & $\begin{array}{l}\text { तै } \\
\stackrel{0}{\sim}\end{array}$ & $\begin{array}{l}\underset{\sim}{N} \\
\stackrel{\sim}{N}\end{array}$ & ָ̊ & $\stackrel{\nabla}{\stackrel{\sim}{\sim}}$ & 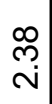 & $\begin{array}{l}\infty \\
\infty \\
0\end{array}$ \\
\hline 훈 & 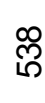 & $\stackrel{m}{\infty}$ & $\stackrel{0}{\stackrel{N}{N}}$ & $\begin{array}{l}\text { के } \\
\text { in }\end{array}$ & 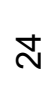 & 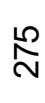 & $R$ & $\frac{n}{m}$ & $\stackrel{m}{0}$ & 10 & $\widetilde{\varrho}$ & $\begin{array}{l}\infty \\
\stackrel{\infty}{\text { N }}\end{array}$ & $\begin{array}{l}\overline{6} \\
\stackrel{+}{\leftarrow}\end{array}$ & $\begin{array}{l}\bar{m} \\
\stackrel{\leftrightarrow}{\sim}\end{array}$ & $\stackrel{\infty}{+}$ & $\stackrel{10}{\stackrel{N}{\sim}}$ & 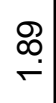 & $\begin{array}{l}\infty \\
0 \\
0\end{array}$ \\
\hline$\stackrel{\bar{\pi}}{\Sigma} \stackrel{\square}{\square}$ & 은 & $\check{\infty}^{\circ}$ & $\stackrel{\infty}{\stackrel{\sim}{\sim}}$ & مْ & ㅇ & 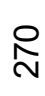 & $\widetilde{\mho}$ & ల్లి & $\begin{array}{l}\infty \\
0 \\
0\end{array}$ & & $\stackrel{\mathcal{V}}{\underset{T}{*}}$ & $\begin{array}{l}\stackrel{8}{0} \\
\stackrel{N}{N}\end{array}$ & $\begin{array}{l}\stackrel{8}{\infty} \\
\stackrel{N}{\sim}\end{array}$ & $\begin{array}{l}\text { గ్ర్ } \\
\text { సై }\end{array}$ & $\checkmark$ & $\stackrel{\stackrel{\varphi}{F}}{F}$ & $\stackrel{\circ}{\stackrel{\leftrightarrow}{r}}$ & ণ্ণি \\
\hline
\end{tabular}

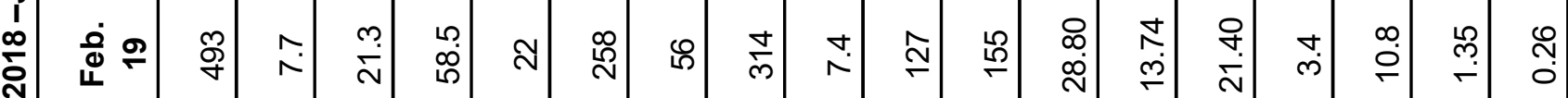

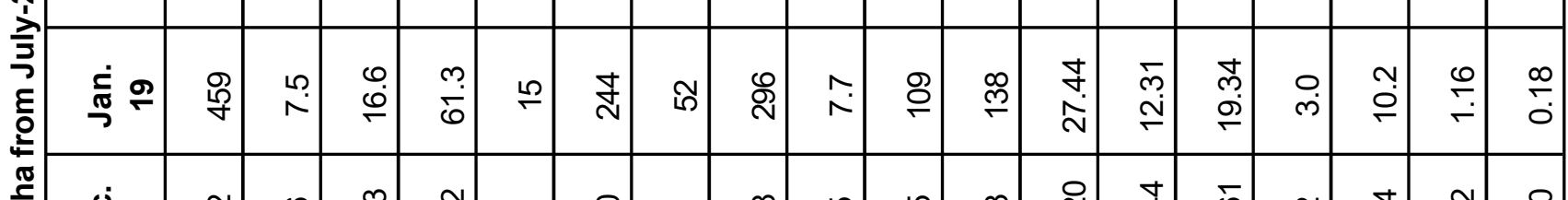

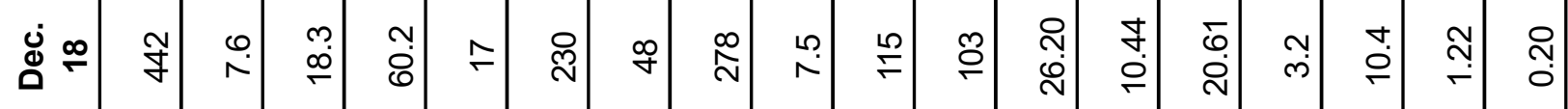

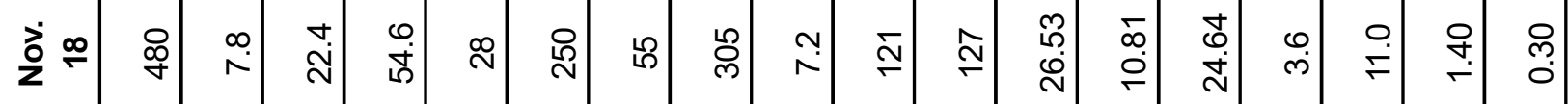

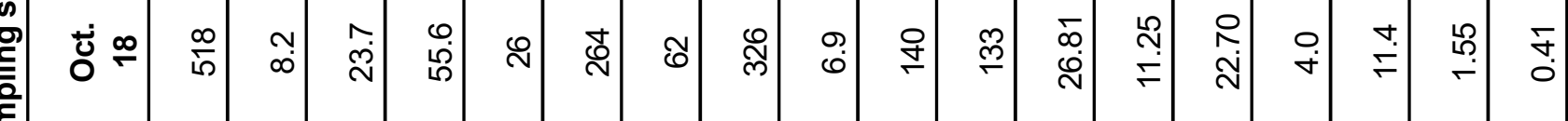

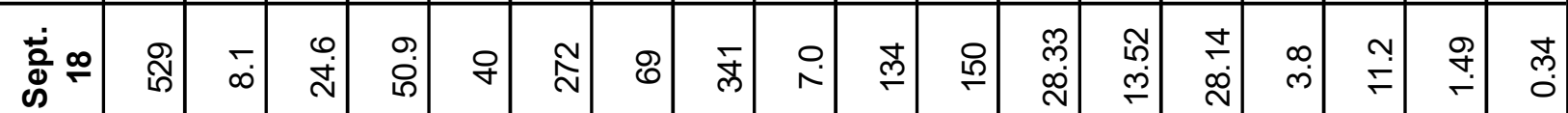

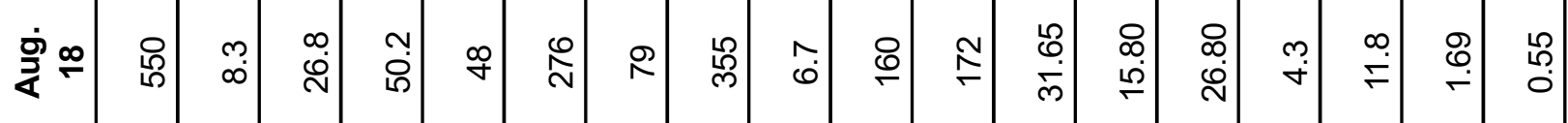

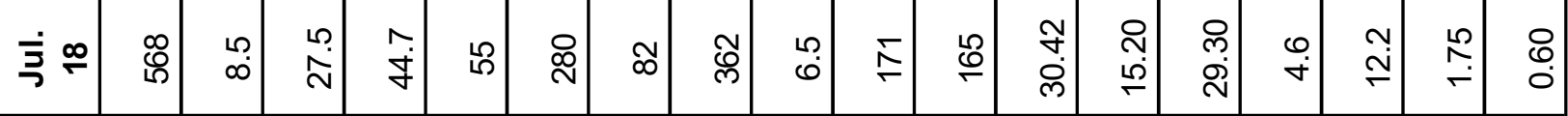

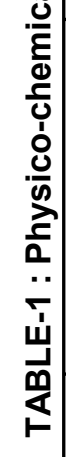

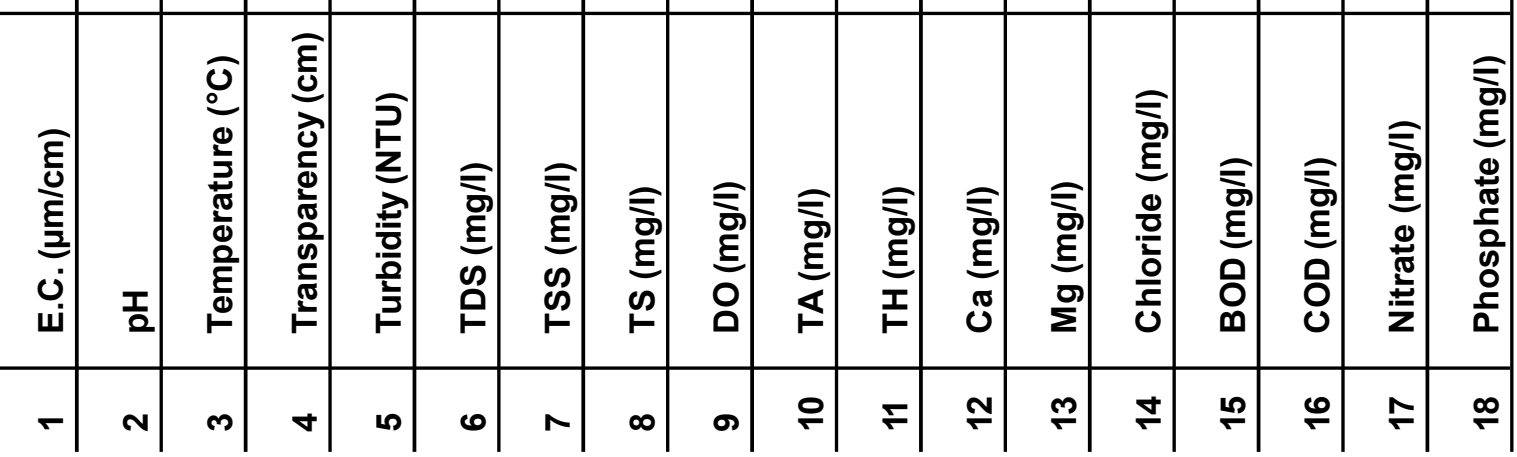




\begin{tabular}{|c|c|c|c|c|c|c|c|c|c|c|c|c|c|c|c|c|c|c|}
\hline 움 & & & & & & & & & & & & & & & & & & - \\
\hline 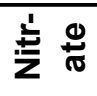 & & & & & & & & & & & & & & & & & - & $\begin{array}{l}8 \\
\stackrel{8}{\circ} \\
0\end{array}$ \\
\hline Оి & & & & & & & & & & & & & & & & - & $\begin{array}{l}\bar{\delta} \\
\\
\end{array}$ & $\begin{array}{l}\hat{\infty} \\
\text { o } \\
0 \\
0\end{array}$ \\
\hline 욤 & & & & & & & & & & & & & & & - & $\begin{array}{l}\text { ஓ. } \\
\text { ه. } \\
\circ\end{array}$ & \begin{tabular}{l}
$\infty$ \\
$\mathscr{8}$ \\
\hdashline \\
0
\end{tabular} & $\begin{array}{l}\text { 吕 } \\
\text { 。 }\end{array}$ \\
\hline$\frac{\dot{0}}{\frac{c}{u}} \frac{0}{\frac{0}{2}}$ & & & & & & & & & & & & & & - & $\begin{array}{l}\stackrel{9}{\circ} \\
\stackrel{2}{0} \\
0\end{array}$ & 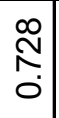 & $\begin{array}{l}\bar{T} \\
0 \\
0\end{array}$ & $\stackrel{\stackrel{ }{N}}{\stackrel{0}{0}}$ \\
\hline$\sum_{\Sigma}^{0}$ & & & & & & & & & & & & & $r$ & \begin{tabular}{l}
\multirow{1}{*}{} \\
6 \\
0 \\
0
\end{tabular} & $\begin{array}{l}\stackrel{R}{N} \\
\hat{o}\end{array}$ & \begin{tabular}{l}
\multirow{D}{0}{} \\
$\stackrel{2}{0}$ \\
0
\end{tabular} & $\begin{array}{l}0 \\
\stackrel{2}{\hat{0}} \\
0\end{array}$ & $\begin{array}{l}\bar{\delta} \\
\infty \\
0 \\
0\end{array}$ \\
\hline తే & & & & & & & & & & & & - & $\begin{array}{l}\stackrel{0}{0} \\
\stackrel{0}{0}\end{array}$ & $\begin{array}{l}\hat{\sigma} \\
\overline{0} \\
0\end{array}$ & $\begin{array}{c}0 \\
\infty \\
\infty \\
0\end{array}$ & $\begin{array}{l}0 \\
\mathscr{N} \\
\infty \\
0\end{array}$ & $\begin{array}{l}\text { No } \\
\infty \\
0 \\
0\end{array}$ & $\begin{array}{c}\infty \\
\infty \\
\infty \\
\infty \\
0\end{array}$ \\
\hline I & & & & & & & & & & & - & $\begin{array}{l}\text { Oे. } \\
\text { O. } \\
\text { O. }\end{array}$ & $\begin{array}{l}\mathscr{8} \\
\stackrel{0}{\circ} \\
\circ\end{array}$ & $\begin{array}{l}\overline{5} \\
0 \\
0 \\
0\end{array}$ & 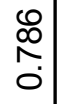 & $\begin{array}{l}\stackrel{+}{0} \\
\stackrel{0}{0}\end{array}$ & $\begin{array}{l}\bar{\alpha} \\
\stackrel{0}{0}\end{array}$ & $\begin{array}{l}0 \\
0 \\
0 \\
0 \\
0\end{array}$ \\
\hline $\mathbb{\digamma}$ & & & & & & & & & & - & $\begin{array}{c}\overline{\dot{\sigma}} \\
\infty \\
\dot{0}\end{array}$ & $\begin{array}{l}\bar{\delta} \\
\infty \\
0 \\
0\end{array}$ & \begin{tabular}{l}
0 \\
\multirow{+}{*}{} \\
0 \\
0
\end{tabular} & $\begin{array}{l}\text { Nิ } \\
\stackrel{0}{0} \\
0\end{array}$ & $\begin{array}{l}\stackrel{L}{~} \\
\text { Oे } \\
0\end{array}$ & $\begin{array}{l}\text { டி } \\
\text { هి } \\
0\end{array}$ & $\begin{array}{l}\mathscr{8} \\
\infty \\
0 \\
0\end{array}$ & $\begin{array}{l}\stackrel{0}{\sigma} \\
0 \\
0\end{array}$ \\
\hline 8 & & & & & & & & & $r$ & $\begin{array}{l}\infty \\
\infty \\
\infty \\
0\end{array}$ & $\begin{array}{l}R \\
R \\
i\end{array}$ & $\begin{array}{l}\text { ले } \\
\infty \\
0 \\
\end{array}$ & $\begin{array}{l}\hat{n} \\
\stackrel{1}{0} \\
\dot{9}\end{array}$ & \begin{tabular}{l}
$\infty$ \\
0 \\
0 \\
\hdashline \\
\hdashline
\end{tabular} & 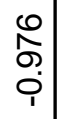 & $\begin{array}{l}\bar{\delta} \\
\text { ه़. } \\
\end{array}$ & 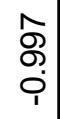 & 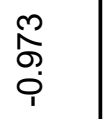 \\
\hline$\stackrel{\mathscr{\rho}}{\vdash}$ & & & & & & & & $r$ & 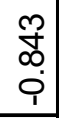 & $\begin{array}{l}8 \\
\infty \\
0 \\
0\end{array}$ & $\begin{array}{l}1 \\
\infty \\
\infty \\
0 \\
0\end{array}$ & $\begin{array}{l}\text { N } \\
\infty \\
0 \\
0\end{array}$ & $\begin{array}{l}\mathbb{T} \\
\infty \\
0 \\
0\end{array}$ & $\begin{array}{c}R \\
0 \\
0 \\
0\end{array}$ & $\begin{array}{l}\hat{D} \\
\infty \\
0 \\
0\end{array}$ & $\begin{array}{c}\infty \\
\infty \\
\infty \\
0 \\
0\end{array}$ & 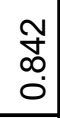 & $\begin{array}{l}\widetilde{1} \\
\infty \\
\infty \\
0 \\
0\end{array}$ \\
\hline$\stackrel{\mathscr{H}}{\mapsto}$ & & & & & & & - & $\begin{array}{l}0 \\
\stackrel{1}{ } \\
0 \\
0\end{array}$ & $\begin{array}{l}\hat{m} \\
0 \\
\infty \\
0 \\
1\end{array}$ & $\begin{array}{l}\infty \\
\sigma \\
\sigma \\
0\end{array}$ & $\begin{array}{l}0 \\
6 \\
\infty \\
\infty \\
0\end{array}$ & $\begin{array}{l}\text { पे } \\
\infty \\
0 \\
0\end{array}$ & $\begin{array}{l}\infty \\
\infty \\
\infty \\
0 \\
0\end{array}$ & $\begin{array}{l}\qquad \\
\infty \\
\infty \\
0 \\
0\end{array}$ & $\begin{array}{l}\mathbb{1} \\
\infty \\
\infty \\
0 \\
0\end{array}$ & $\begin{array}{l} \\
\bigotimes \\
\infty \\
0 \\
0\end{array}$ & $\begin{array}{l}\hat{N} \\
\infty \\
0 \\
0\end{array}$ & $\begin{array}{l}\hat{N} \\
\infty \\
0 \\
0\end{array}$ \\
\hline$\stackrel{\mathscr{P}}{\underline{R}}$ & & & & & & - & $\begin{array}{l}\text { న్ } \\
\text { Oे. }\end{array}$ & $\begin{array}{l}\hat{0} \\
\stackrel{0}{\circ} \\
0\end{array}$ & $\begin{array}{c}\text { N } \\
0 \\
0 \\
i\end{array}$ & $\begin{array}{l}-1 \\
\infty \\
0 \\
0\end{array}$ & $\begin{array}{l}0 \\
\infty \\
\infty \\
0\end{array}$ & $\begin{array}{l}\infty \\
\stackrel{1}{N} \\
0\end{array}$ & $\begin{array}{l}\mathbb{Z} \\
\infty \\
0 \\
0\end{array}$ & $\begin{array}{l}\bar{\infty} \\
\infty \\
0 \\
0\end{array}$ & $\begin{array}{l}0 \\
\infty \\
\infty \\
0\end{array}$ & $\begin{array}{l}8 \\
\mathscr{0} \\
\infty \\
0 \\
0\end{array}$ & $\begin{array}{c}\bar{్} \\
\infty \\
0 \\
0\end{array}$ & $\begin{array}{l}\mathscr{0} \\
\infty \\
0 \\
0\end{array}$ \\
\hline 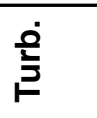 & & & & & - & \begin{tabular}{l}
\multirow{0}{0}{} \\
0 \\
0
\end{tabular} & $\begin{array}{l}\Re \\
\infty \\
0 \\
0\end{array}$ & 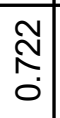 & 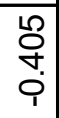 & 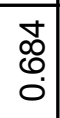 & $\begin{array}{l}\Re \\
0 \\
0 \\
0 \\
0\end{array}$ & $\begin{array}{l}\text { \& } \\
\text { L } \\
0\end{array}$ & $\begin{array}{l}\tilde{\delta} \\
0 \\
0\end{array}$ & $\begin{array}{l}\bar{q} \\
0 \\
0\end{array}$ & 宫 & 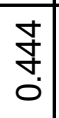 & $\begin{array}{l}\infty \\
\infty \\
m \\
0 \\
0\end{array}$ & $\underset{0}{\bar{g}}$ \\
\hline 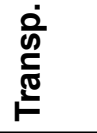 & & & & $r$ & $\begin{array}{l}\overline{\hat{N}} \\
0 \\
0 \\
1\end{array}$ & $\begin{array}{l}\hat{0} \\
0 \\
0 \\
1\end{array}$ & $\begin{array}{l}\mathscr{0} \\
\mathbb{0} \\
0 \\
i \\
1\end{array}$ & $\begin{array}{l}0 \\
\stackrel{N}{N} \\
0 \\
\dot{1}\end{array}$ & \begin{tabular}{l}
$\stackrel{8}{0}$ \\
\multirow{+}{*}{} \\
0
\end{tabular} & $\begin{array}{l}\mathbf{N} \\
\mathbf{N} \\
0 \\
\mathbf{1}\end{array}$ & $\begin{array}{l}0 \\
0 \\
0 \\
0 \\
0 \\
\end{array}$ & $\begin{array}{l}0 \\
10 \\
0 \\
0 \\
1\end{array}$ & $\begin{array}{l}N \\
N \\
0 \\
0 \\
1\end{array}$ & $\begin{array}{c}\bar{n} \\
\infty \\
0 \\
0 \\
1\end{array}$ & \begin{tabular}{l}
0 \\
\multirow{1}{0}{} \\
0 \\
$i$ \\
1
\end{tabular} & 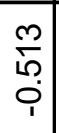 & 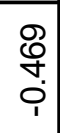 & 훙 \\
\hline$\stackrel{\stackrel{\varrho}{\varepsilon}}{\stackrel{\Xi}{\varrho}}$ & & & - & $\begin{array}{l}0 \\
1 \\
0 \\
0 \\
1\end{array}$ & 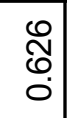 & $\begin{array}{c}\hat{\emptyset} \\
\text { Ŏ }\end{array}$ & ণ্ & $\begin{array}{l}0 \\
\stackrel{+}{\sigma} \\
\stackrel{0}{0}\end{array}$ & 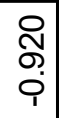 & $\begin{array}{l}\infty \\
\stackrel{0}{\circ} \\
\text { ه़ }\end{array}$ & $\begin{array}{l}\infty \\
\stackrel{2}{1} \\
0\end{array}$ & $\begin{array}{l}0 \\
\stackrel{0}{0} \\
0\end{array}$ & 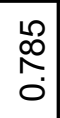 & $\begin{array}{l}\mathscr{1} \\
\varnothing \\
\infty \\
0\end{array}$ & $\begin{array}{l}L \\
\mathscr{8} \\
0 \\
0\end{array}$ & 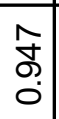 & $\stackrel{m}{\sigma}$ & $\begin{array}{l}\text { fo } \\
\text { ơ }\end{array}$ \\
\hline 工ٓ & & - & $\begin{array}{l}\hat{\imath} \\
\stackrel{\hat{\rho}}{0} \\
0\end{array}$ & $\begin{array}{l}\text { गे } \\
0 \\
0 \\
1\end{array}$ & 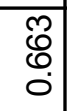 & 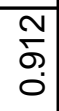 & 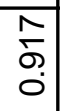 & 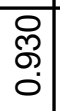 & $\begin{array}{l}\hat{0} \\
\text { o } \\
0 \\
1\end{array}$ & $\begin{array}{l}0 \\
\text { مొ } \\
0 \\
0\end{array}$ & $\begin{array}{l}9 \\
0 \\
0 \\
0\end{array}$ & $\begin{array}{l}m \\
\stackrel{m}{N} \\
0\end{array}$ & $\begin{array}{l} \\
\stackrel{5}{0} \\
0\end{array}$ & $\begin{array}{l}10 \\
0 \\
0 \\
0\end{array}$ & 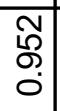 & $\begin{array}{l}\infty \\
\dot{\sigma} \\
0\end{array}$ & $\begin{array}{l}\Lambda \\
\infty \\
\infty \\
0\end{array}$ & $\begin{array}{l}\widetilde{N} \\
\text { Oे } \\
0\end{array}$ \\
\hline نُ & - & $\begin{array}{l}\bar{\sigma} \\
\stackrel{0}{0}\end{array}$ & $\begin{array}{l}\hat{S} \\
\stackrel{\sigma}{0} \\
\dot{0}\end{array}$ & $\begin{array}{l}\hat{N} \\
\stackrel{2}{0} \\
i \\
i\end{array}$ & $\begin{array}{l}\stackrel{1}{0} \\
\hat{0} \\
0\end{array}$ & 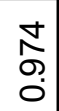 & $\begin{array}{l}9 \\
0 \\
0 \\
0\end{array}$ & $\begin{array}{l}\mathscr{\Omega} \\
\text { S. } \\
0\end{array}$ & $\begin{array}{l}0 \\
0 \\
\infty \\
0 \\
0\end{array}$ & 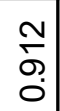 & $\begin{array}{l}\overline{0} \\
\infty \\
0\end{array}$ & $\begin{array}{c}N \\
\stackrel{N}{\infty} \\
0\end{array}$ & 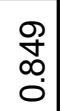 & $\begin{array}{l}0 \\
\infty \\
\infty \\
0\end{array}$ & 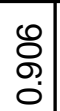 & $\begin{array}{c}0 \\
\infty \\
\infty \\
0\end{array}$ & $\begin{array}{l}\text { g } \\
\text { D } \\
0\end{array}$ & $\begin{array}{l}\mathscr{D} \\
\infty \\
\infty \\
0\end{array}$ \\
\hline 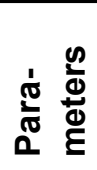 & نे & $\frac{\tau}{2}$ & $\begin{array}{l}\stackrel{0}{E} \\
\stackrel{\Xi}{\varrho} \\
-1\end{array}$ & $\begin{array}{l}\dot{0} \\
\frac{0}{\pi} \\
\frac{2}{\sqrt{n}} \\
=\end{array}$ & $\begin{array}{l}\frac{7}{7} \\
\frac{0}{0} \\
\frac{0}{2} \\
\frac{1}{3}\end{array}$ & 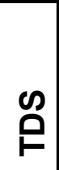 & $\begin{array}{c}\mathscr{D} \\
\stackrel{1}{\circ}\end{array}$ & $\mathscr{\rho}$ & ৪ & 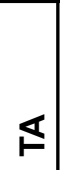 & $I$ & ర్ & $\sum^{\infty}$ & $\begin{array}{l}\frac{0}{0} \\
\frac{0}{\frac{0}{2}} \\
\frac{\mathrm{c}}{\mathrm{U}}\end{array}$ & Оి & ర్ర & 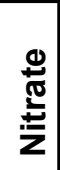 & 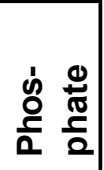 \\
\hline
\end{tabular}




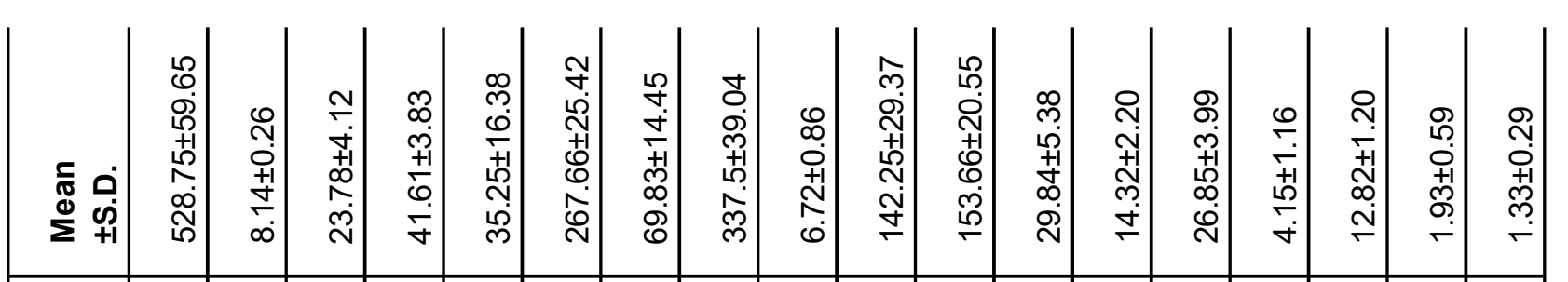

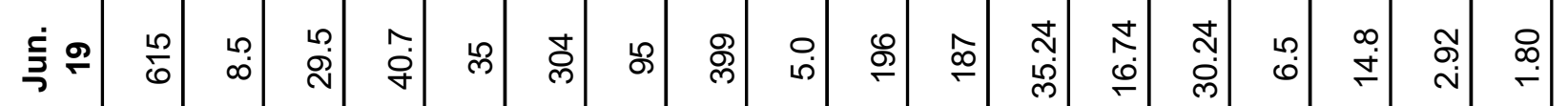

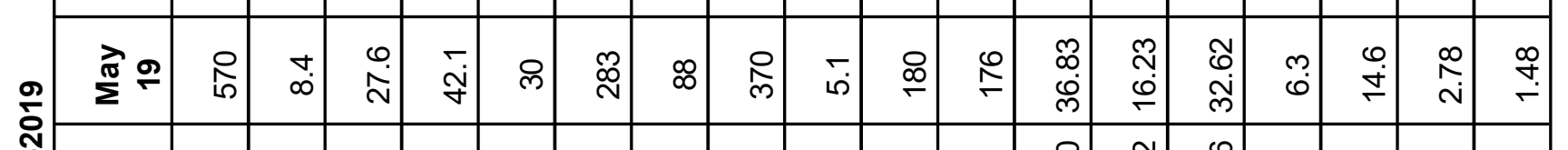

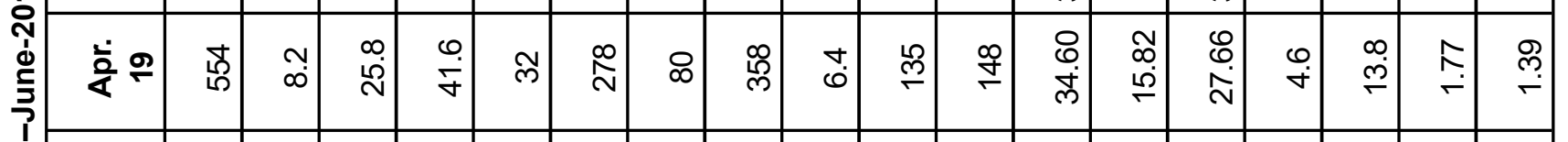

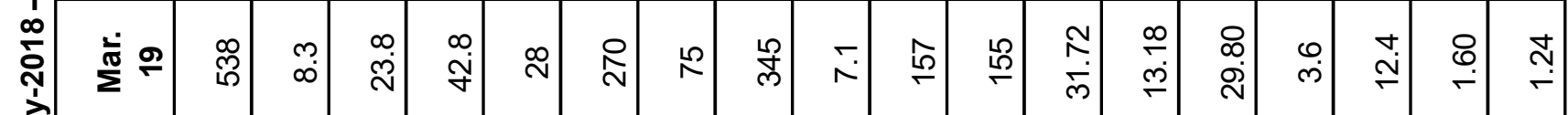

길

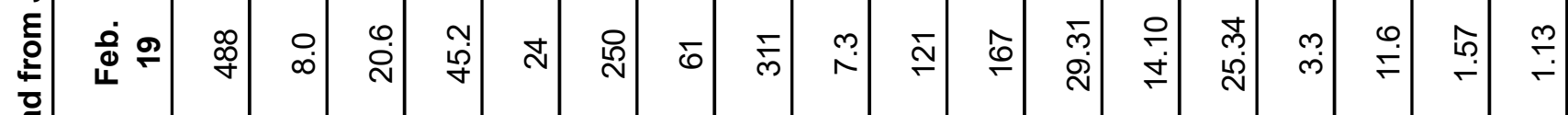

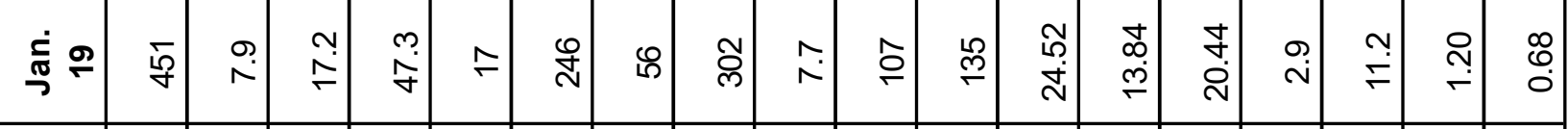

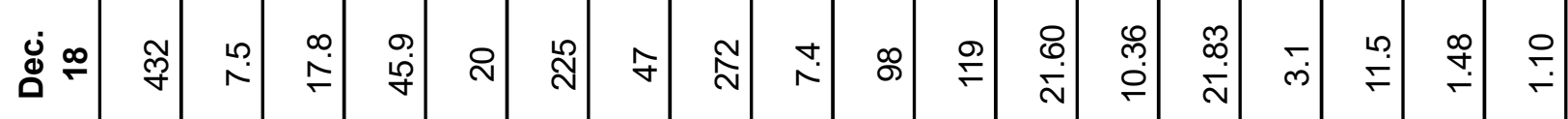

중

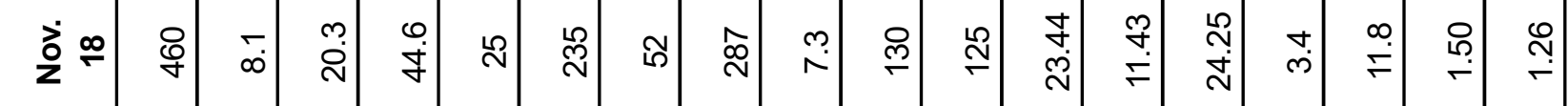

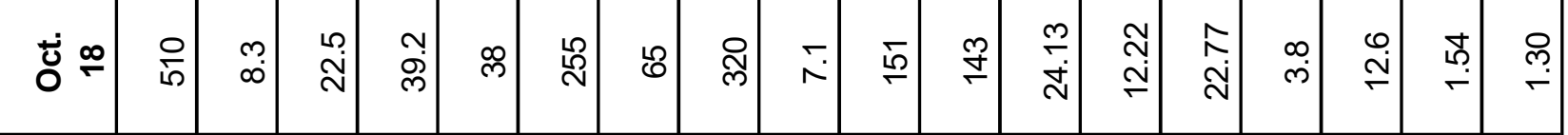

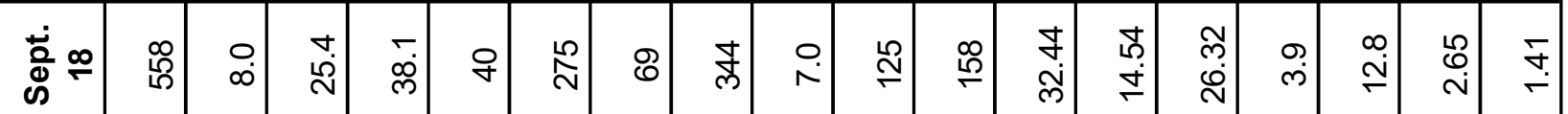

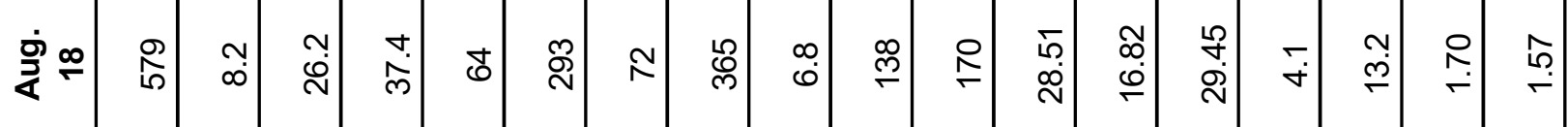

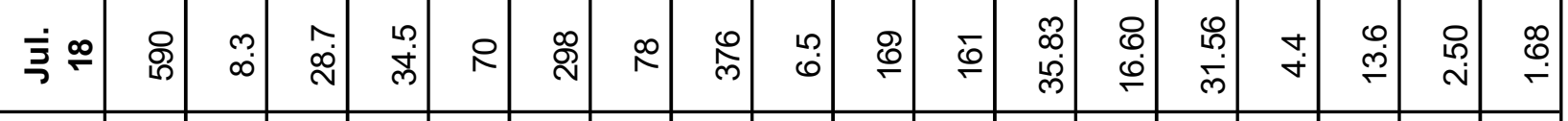

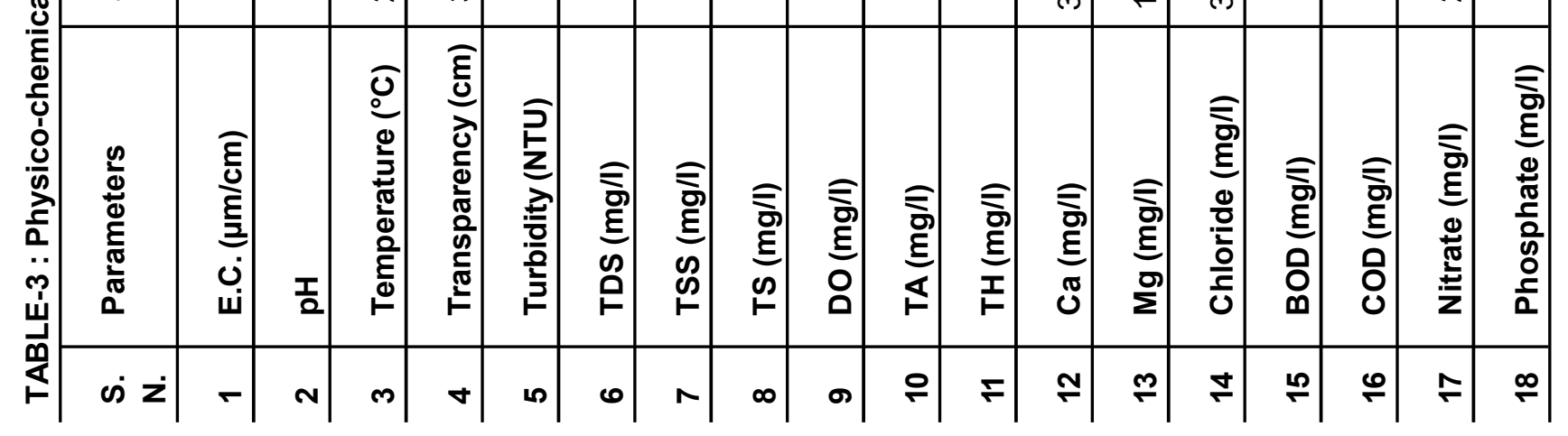




\begin{tabular}{|c|c|c|c|c|c|c|c|c|c|c|c|c|c|c|c|c|c|c|}
\hline $\begin{array}{l}0 \\
\stackrel{0}{0} \\
\frac{2}{\alpha}\end{array}$ & & & & & & & & & & & & & & & & & & $r$ \\
\hline 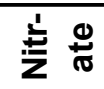 & & & & & & & & & & & & & & & & & $r$ & $\begin{array}{l}\text { N } \\
\text { 足 }\end{array}$ \\
\hline Оᄋ & & & & & & & & & & & & & & & & $r$ & $\begin{array}{c}\stackrel{L}{0} \\
\infty \\
0 \\
0\end{array}$ & $\begin{array}{l}\text { స్రి } \\
\infty \\
0 \\
0\end{array}$ \\
\hline Оి & & & & & & & & & & & & & & & - & $\begin{array}{l}\bar{\sigma} \\
\text { م. } \\
0\end{array}$ & $\begin{array}{l}\mathscr{L} \\
\infty \\
\infty \\
0\end{array}$ & $\begin{array}{l}\frac{N}{N} \\
\stackrel{0}{0}\end{array}$ \\
\hline$\frac{\dot{0}}{\frac{1}{0}} \frac{0}{2}$ & & & & & & & & & & & & & & - & $\begin{array}{l}\stackrel{g}{+} \\
\stackrel{0}{0}\end{array}$ & $\begin{array}{c}\stackrel{1}{N} \\
\infty \\
0 \\
0\end{array}$ & $\begin{array}{l}\stackrel{L}{N} \\
\stackrel{0}{0}\end{array}$ & $\begin{array}{l}8 \\
\infty \\
0 \\
0\end{array}$ \\
\hline$\sum^{\infty}$ & & & & & & & & & & & & & $r$ & $\begin{array}{l}\bar{\phi} \\
\stackrel{0}{0}\end{array}$ & $\begin{array}{l}\hat{0} \\
0 \\
0 \\
0\end{array}$ & $\begin{array}{l}N \\
N \\
0\end{array}$ & 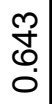 & $\begin{array}{l}\hat{\mathscr{O}} \\
0 \\
0\end{array}$ \\
\hline రే & & & & & & & & & & & & - & $\begin{array}{c}\stackrel{2}{N} \\
\infty \\
0\end{array}$ & $\begin{array}{c}\infty \\
\infty \\
\infty \\
0\end{array}$ & $\begin{array}{l}8 \\
\stackrel{0}{0} \\
0\end{array}$ & $\begin{array}{c}\stackrel{2}{N} \\
\infty \\
0\end{array}$ & $\begin{array}{l}\infty \\
\stackrel{2}{0} \\
0\end{array}$ & $\begin{array}{l}\bar{T} \\
0 \\
0\end{array}$ \\
\hline$I$ & & & & & & & & & & & - & $\begin{array}{l}2 \\
\stackrel{2}{0} \\
0\end{array}$ & $\begin{array}{l}\mathbb{Z} \\
\infty \\
0 \\
0\end{array}$ & $\begin{array}{l}0 \\
0 \\
\\
0\end{array}$ & \begin{tabular}{l|}
\multirow{2}{*}{} \\
$\stackrel{0}{0}$ \\
0
\end{tabular} & 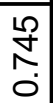 & $\begin{array}{l}0 \\
\stackrel{0}{N} \\
0\end{array}$ & $\begin{array}{l}10 \\
0 \\
0 \\
0\end{array}$ \\
\hline $\mathbb{\leftarrow}$ & & & & & & & & & & - & $\begin{array}{l}\stackrel{p}{N} \\
\stackrel{0}{0}\end{array}$ & $\begin{array}{l}\stackrel{+}{N} \\
0\end{array}$ & 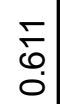 & 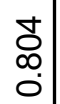 & $\begin{array}{l}\hat{L} \\
\infty \\
0\end{array}$ & $\begin{array}{l}0 \\
\stackrel{1}{0} \\
\infty \\
0\end{array}$ & $\begin{array}{l}\hat{N} \\
0 \\
0\end{array}$ & $\frac{N}{N}$ \\
\hline 8 & & & & & & & & & $r$ & $\begin{array}{c}\bar{n} \\
\infty \\
0 \\
0\end{array}$ & 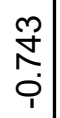 & $\begin{array}{l}0 \\
0 \\
0 \\
0\end{array}$ & 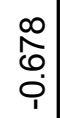 & 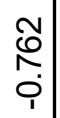 & $\begin{array}{l}\hat{8} \\
\text { م. } \\
\text { i }\end{array}$ & 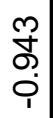 & $\begin{array}{l}m \\
\stackrel{0}{\infty} \\
\dot{1}\end{array}$ & 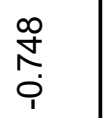 \\
\hline$\stackrel{\mathscr{P}}{\leftarrow}$ & & & & & & & & - & $\begin{array}{l}2 \\
\infty \\
\infty \\
0 \\
1\end{array}$ & $\begin{array}{l}\mathscr{M} \\
\infty \\
0 \\
0\end{array}$ & $\begin{array}{l}\infty \\
0 \\
\infty \\
0\end{array}$ & $\begin{array}{l}\infty \\
\infty \\
\infty \\
0\end{array}$ & $\begin{array}{l}\hat{\sigma} \\
0 \\
0\end{array}$ & $\begin{array}{l}\infty \\
0 \\
\infty \\
0\end{array}$ & $\begin{array}{l}n \\
\infty \\
0 \\
0\end{array}$ & $\begin{array}{l}\text { 응 } \\
\text { ㅇ. }\end{array}$ & 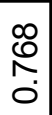 & $\begin{array}{l}\hat{\circ} \\
\infty \\
0\end{array}$ \\
\hline$\stackrel{\mathscr{S}}{\leftarrow}$ & & & & & & & - & $\begin{array}{l}\mathscr{8} \\
\mathscr{0} \\
0\end{array}$ & $\begin{array}{l}9 \\
\text { O. } \\
0 \\
0\end{array}$ & $\begin{array}{c}\mathscr{m} \\
\infty \\
\infty \\
0 \\
0\end{array}$ & 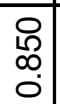 & 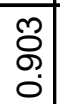 & $\begin{array}{c}\hat{N} \\
\infty \\
0 \\
0\end{array}$ & $\begin{array}{l}\tilde{W} \\
\infty \\
0 \\
0\end{array}$ & $\begin{array}{l}\infty \\
0 \\
\infty \\
0 \\
0\end{array}$ & 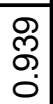 & 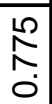 & $\begin{array}{l}\stackrel{\mathscr{R}}{R} \\
\stackrel{0}{0}\end{array}$ \\
\hline$\stackrel{\infty}{\mathscr{1}}$ & & & & & & - & $\begin{array}{l}\text { 응 } \\
\text { o. }\end{array}$ & $\begin{array}{l}\infty \\
\infty \\
O \\
0\end{array}$ & $\begin{array}{l}\bar{n} \\
\hat{p} \\
i\end{array}$ & $\begin{array}{l}\hat{N} \\
\hat{0}\end{array}$ & $\begin{array}{c}9 \\
\$ \\
0 \\
0\end{array}$ & $\begin{array}{l}\overline{1} \\
\infty \\
0 \\
0\end{array}$ & $\begin{array}{l}\stackrel{2}{2} \\
\text { Oे }\end{array}$ & $\begin{array}{c}9 \\
\$ \\
0 \\
0\end{array}$ & \begin{tabular}{l}
\multirow{J}{*}{} \\
0 \\
0
\end{tabular} & $\begin{array}{c}0 \\
0 \\
0 \\
0\end{array}$ & $\begin{array}{l}\stackrel{\rho}{\Omega} \\
\stackrel{0}{0}\end{array}$ & 京 \\
\hline 咅 & & & & & $r$ & $\begin{array}{l}0 \\
2 \\
0\end{array}$ & $\begin{array}{c}\text { ஓ్ } \\
\text { ○. }\end{array}$ & $\begin{array}{l}2 \\
0 \\
0 \\
0\end{array}$ & 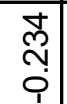 & 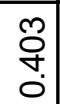 & 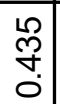 & 웜 & $\begin{array}{l}0 \\
8 \\
0 \\
0\end{array}$ & $\begin{array}{l}0 \\
0 \\
0 \\
0 \\
0\end{array}$ & 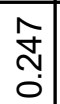 & 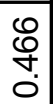 & ळ్ల & $\begin{array}{l}\infty \\
8 \\
0 \\
0\end{array}$ \\
\hline 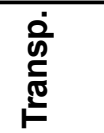 & & & & $r$ & 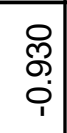 & 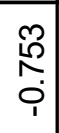 & $\begin{array}{l}\text { N్} \\
\hat{W} \\
0 \\
\end{array}$ & $\begin{array}{l}0 \\
0 \\
\vdots \\
0 \\
1\end{array}$ & 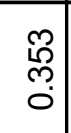 & \begin{tabular}{l}
\multirow{N}{*}{} \\
$\hat{0}$ \\
0 \\
1
\end{tabular} & 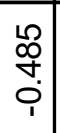 & $\begin{array}{l}\bar{N} \\
\text { స్ } \\
0 \\
\end{array}$ & $\begin{array}{l}\text { 吕 } \\
0 \\
0 \\
0 \\
1\end{array}$ & $\begin{array}{l}\infty \\
0 \\
0 \\
0 \\
\\
1\end{array}$ & $\begin{array}{l}\hat{N} \\
\hat{m} \\
0 \\
\end{array}$ & $\begin{array}{l}\hat{\circ} \\
0 \\
0 \\
0\end{array}$ & $\begin{array}{l}\Re \\
0 \\
0 \\
0 \\
1\end{array}$ & 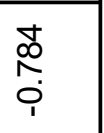 \\
\hline 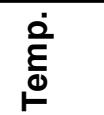 & & & - & 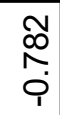 & $\begin{array}{l}\overline{\hat{0}} \\
0 \\
0\end{array}$ & 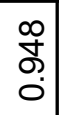 & $\begin{array}{l}\infty \\
\delta \\
\delta\end{array}$ & $\begin{array}{l}\hat{ٌ} \\
0 \\
0\end{array}$ & $\begin{array}{l}0 \\
\infty \\
0 \\
0 \\
1\end{array}$ & $\begin{array}{l}0 \\
0 \\
0 \\
0\end{array}$ & $\begin{array}{c}0 \\
\varnothing \\
\infty \\
0\end{array}$ & $\begin{array}{l}\tilde{N} \\
\infty \\
0 \\
0\end{array}$ & $\begin{array}{c}\mathbb{N} \\
\infty \\
0 \\
0\end{array}$ & $\begin{array}{l}\text { ৪. } \\
\text { ৪. } \\
\circ\end{array}$ & $\begin{array}{c}\stackrel{L}{0} \\
\infty \\
0 \\
0\end{array}$ & $\begin{array}{l}0 \\
\mathscr{O} \\
0 \\
0\end{array}$ & $\begin{array}{l}\stackrel{+}{W} \\
\infty \\
0\end{array}$ & O্. \\
\hline I & & - & $\begin{array}{l}\Omega \\
\stackrel{2}{0} \\
0\end{array}$ & 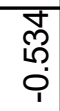 & 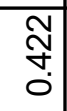 & $\begin{array}{l} \\
0 \\
0 \\
0\end{array}$ & \begin{tabular}{l}
$\mathcal{N}$ \\
\multirow{1}{*}{} \\
0 \\
0
\end{tabular} & 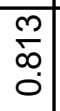 & $\begin{array}{l}8 \\
\stackrel{0}{0} \\
\text { i. }\end{array}$ & 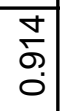 & $\begin{array}{l}N \\
\\
0\end{array}$ & $\begin{array}{l}1 \\
0 \\
0 \\
0\end{array}$ & $\begin{array}{l}0 \\
0 \\
0 \\
0\end{array}$ & 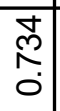 & $\begin{array}{l}\stackrel{d}{N} \\
\hat{0}\end{array}$ & $\begin{array}{l}8 \\
0 \\
0\end{array}$ & 荀 & $\begin{array}{l}\text { No } \\
0 \\
0\end{array}$ \\
\hline نُ & - & $\begin{array}{l}\mathscr{R} \\
\stackrel{2}{0} \\
0\end{array}$ & $\begin{array}{l}\bar{\infty} \\
\stackrel{0}{0} \\
0\end{array}$ & $\begin{array}{l}0 \\
\stackrel{D}{1} \\
0 \\
0\end{array}$ & $\begin{array}{l}1 \\
o \\
0 \\
0\end{array}$ & $\begin{array}{l}\mathscr{0} \\
\mathscr{0} \\
O \\
0\end{array}$ & $\begin{array}{l}\stackrel{+}{\sigma} \\
\stackrel{0}{\circ}\end{array}$ & 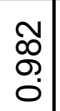 & 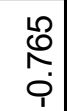 & $\begin{array}{c}0 \\
0 \\
0 \\
0\end{array}$ & $\begin{array}{c}\tilde{W} \\
\infty \\
0 \\
0\end{array}$ & $\begin{array}{l}\nwarrow \\
\varnothing \\
0 \\
0\end{array}$ & $\begin{array}{l}\infty \\
0 \\
\infty \\
0\end{array}$ & $\begin{array}{c}\bar{T} \\
\infty \\
0 \\
0\end{array}$ & $\begin{array}{l}8 \\
\stackrel{0}{0} \\
0\end{array}$ & $\begin{array}{c}\infty \\
\varnothing \\
\infty \\
0\end{array}$ & $\begin{array}{l}\infty \\
\infty \\
\hat{0} \\
0\end{array}$ & $\begin{array}{l}\mathscr{m} \\
\infty \\
0 \\
0\end{array}$ \\
\hline 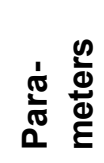 & نُ & $\frac{T}{2}$ & $\begin{array}{l}\dot{\grave{g}} \\
\stackrel{\vec{d}}{\sigma}\end{array}$ & $\begin{array}{l}\frac{\dot{0}}{0} \\
\frac{2}{\pi} \\
\frac{0}{2}\end{array}$ & $\begin{array}{l}\frac{7}{7} \\
\frac{0}{0} \\
\frac{0}{2} \\
\frac{1}{3}\end{array}$ & $\underset{c}{\infty}$ & $\mathscr{\mathscr { S }}$ & 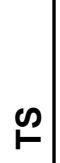 & ৪ & 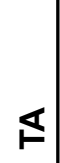 & $I$ & తే & $\sum^{\infty}$ & $\begin{array}{l}\frac{0}{0} \\
\frac{0}{\frac{0}{2}} \\
\frac{\mathrm{c}}{\mathrm{U}}\end{array}$ & Оి & Оิ & 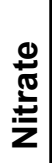 & 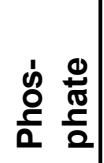 \\
\hline
\end{tabular}


hours in each month throught the year. Airtight plastic containers were used to collect samples from both lentic as well as lotic sampling sites. Some sensitive and unstable parameters were tested at the sampling sites, while other parameters examined at the departmental laboratory of Bipin Bihari College, Jhansi with the help of

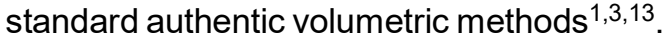

\section{Observation}

During the working, many ups and downs were observed in the water quality parameters of Betwa River. Due to environmental fluctuating conditions, during summer period most of the water parameters increased while other parameters decreased in winter because environmental condition acts as a driving catalyst for natural inland water (Tables 1-3).

\section{Result and Discussion}

\section{Electrical conductivity, Temperature and $\mathrm{pH}$}

Electric conductivity is representative parameter of overall ionic potential present in aquatic body. The conductivity range (Fig.1) of lotic zone was recorded maximum in June $(580 \mu \mathrm{m} / \mathrm{cm})$ and minimum in December $(442 \mu \mathrm{m} / \mathrm{cm})$ with their mean and standard deviation range (518.66 \pm 43.47$)$, while conductivity range of lotic zone was recorded maximum also in June (615 $\mu \mathrm{m} / \mathrm{cm})$ and minimum in December $(432 \mu \mathrm{m} / \mathrm{cm})$ with their mean and standard deviation range $(528.75 \pm 59.65)$. The rapid increase in electric conductivity indicates greater amount of ionic concentration present in aquatic body ${ }^{13}$.

Temperature is the prime regulator parameter for every aquatic life. Water temperature range (Fig.3) of lotic zone was recorded maximum in June $\left(29.7^{\circ} \mathrm{C}\right)$ and minimum in January $\left(16.6^{\circ} \mathrm{C}\right)$ with their mean and standard deviation range $(24.37 \pm 4.14)$, while water temperature range of lentic zone was recorded maximum also in June $\left(29.5^{\circ} \mathrm{C}\right)$ and minimum in January $\left(17.2^{\circ} \mathrm{C}\right)$ with their mean and standard deviation range $(23.78 \pm 4.12)$. It shows negative correlation with dissolved oxygen ${ }^{16}$.

The $\mathrm{pH}$ range (Fig.2) of lotic zone was recorded maximum in May (8.6) and minimum in January (7.5) with their mean and standard deviation range $(8.09 \pm 0.36)$, while $\mathrm{pH}$ range of lentic zone was recorded maximum in June (8.5) and minimum in December (7.5) with their mean and standard deviation range $(8.14 \pm 0.26)$. The $\mathrm{pH}$ shows positive correlation with electrical conductivity, alkalinity and chloride ${ }^{14}$.

\section{Transparency and Turbidity}

The transparency range (Fig.4) of lotic zone was recorded maximum in January $(61.3 \mathrm{~cm})$ and minimum in July (44.7c.m) with their mean and standard deviation range (54.51 \pm 4.97$)$, while transparency range of lentic zone was recorded maximum in January ( $47.3 \mathrm{c.m}$ ) and minimum in July $(34.5 \mathrm{c} . \mathrm{m})$ with their mean and standard deviation range ( $41.61 \pm 3.83)$. The turbidity range of lotic zone was recorded maximum in July ( 55 NTU) and minimum in January (15 NTU) with their mean and standard deviation range $(30 \pm 12.38)$, while turbidity range of lotic zone was recorded maximum in July (70 NTU) and minimum in January (17 NTU) with mean and standard deviation range $(35.25 \pm 16.38)$. In our findings both these parameters are inversely correlated ${ }^{2,7}$.

\section{TDS, TSS and TS}

The TDS range (Fig.6) of lotic zone was recorded maximum in June $(285 \mathrm{mg} / \mathrm{l})$ and minimum in December $(230 \mathrm{mg} / \mathrm{l})$ with their mean and standard deviation range (265.33 \pm 16.74$)$, while TDS range of lotic zone was recorded maximum in June (304 mg/l) and minimum in December $(225 \mathrm{mg} /)$ with their mean and standard deviation range (267.66 \pm 25.42 ). The TSS range (Fig.7) of lotic zone was recorded maximum in June $(85 \mathrm{mg} / \mathrm{l})$ and minimum in December $(48 \mathrm{mg} / \mathrm{l})$ with their mean and standard deviation range $(66.5 \pm 12.52)$, while TSS range of lentic zone was recorded maximum in June $(95 \mathrm{mg} / \mathrm{l})$ and minimum in December $(47 \mathrm{mg} / \mathrm{l})$ with their mean and standard deviation range $(69.83 \pm 14.45)$. The TS range (Fig.8) of lotic zone was recorded maximum in June (370 $\mathrm{mg} / \mathrm{l})$ and minimum in December $(278 \mathrm{mg} / \mathrm{l})$ with their mean and standard deviation range $(331.83 \pm 28.76)$, while TS range of lentic zone was recorded maximum in June (399 $\mathrm{mg} / \mathrm{l})$ and minimum in December $(272 \mathrm{mg} / \mathrm{l})$ with mean and standard deviation range (337.5 \pm 39.04$)$.

\section{Dissolved Oxygen}

Dissolved oxygen is most fundamental driver parameter for regulating the all activities of aquatic biodiversity. The dissolved oxygen range (Fig.9) of lotic zone was recorded maximum in January $(7.7 \mathrm{mg} / \mathrm{l})$ and minimum in May ( $5.4 \mathrm{mg} / \mathrm{l})$ with their mean and standard deviation range $(6.76 \pm 0.68)$, while dissolved oxygen range of lentic zone was recorded maximum also in January $(7.7 \mathrm{mg} / \mathrm{l})$ and minimum in June $(5.0 \mathrm{mg} / \mathrm{l})$ with their mean and standard deviation range $(6.72 \pm 0.86)$. In our findings dissolved oxygen is inversely correlated with temperature because solubility of gases decreases as temperature rises ${ }^{11}$.

\section{TA and TH}

The total alkalinity range (Fig.10) of lotic zone was recorded maximum in May $(181 \mathrm{mg} / \mathrm{l})$ and minimum in January $(109 \mathrm{mg} / \mathrm{l})$ with their mean and standard deviation range (141.58 \pm 22.74$)$, while total alkalinity range of lentic zone was recorded maximum in June $(196 \mathrm{mg} / \mathrm{l})$ and minimum in December $(98 \mathrm{mg} / \mathrm{l})$ with their mean and 

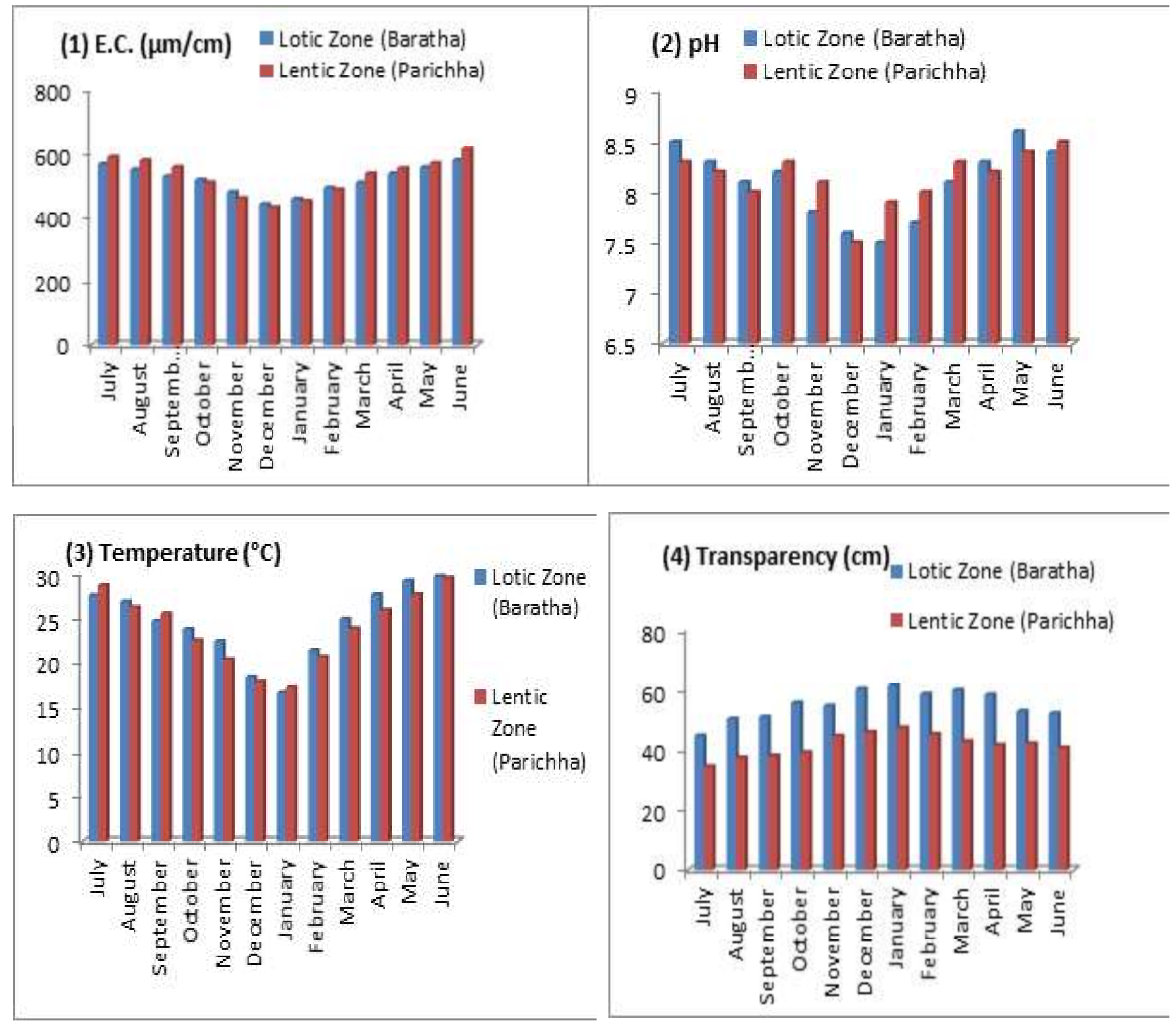

(5) Turbidity (NTU)

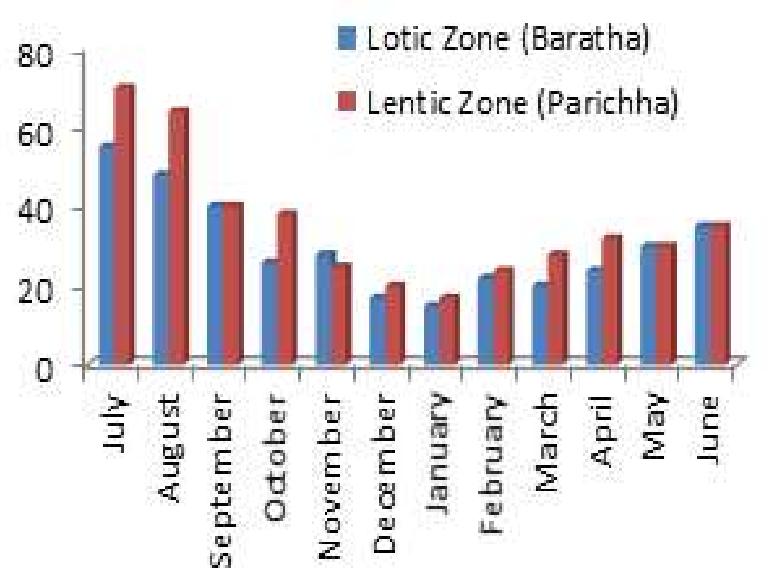

(6) TDS (mg/l) Lotic Zone (Baratha)

- Lentic Zone (Parichha)

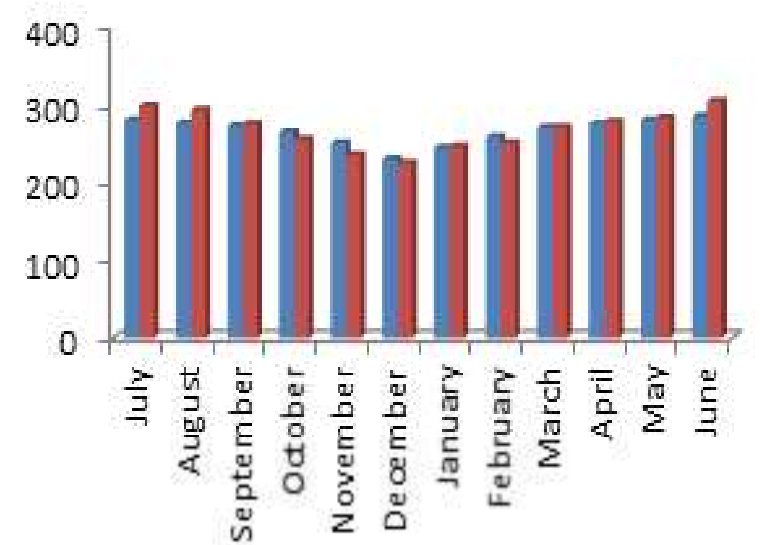

Figs : 1,2,3,4,5 and 6, comparative presentation of physico-chemical parameters of Lotic and Lentic Zones 


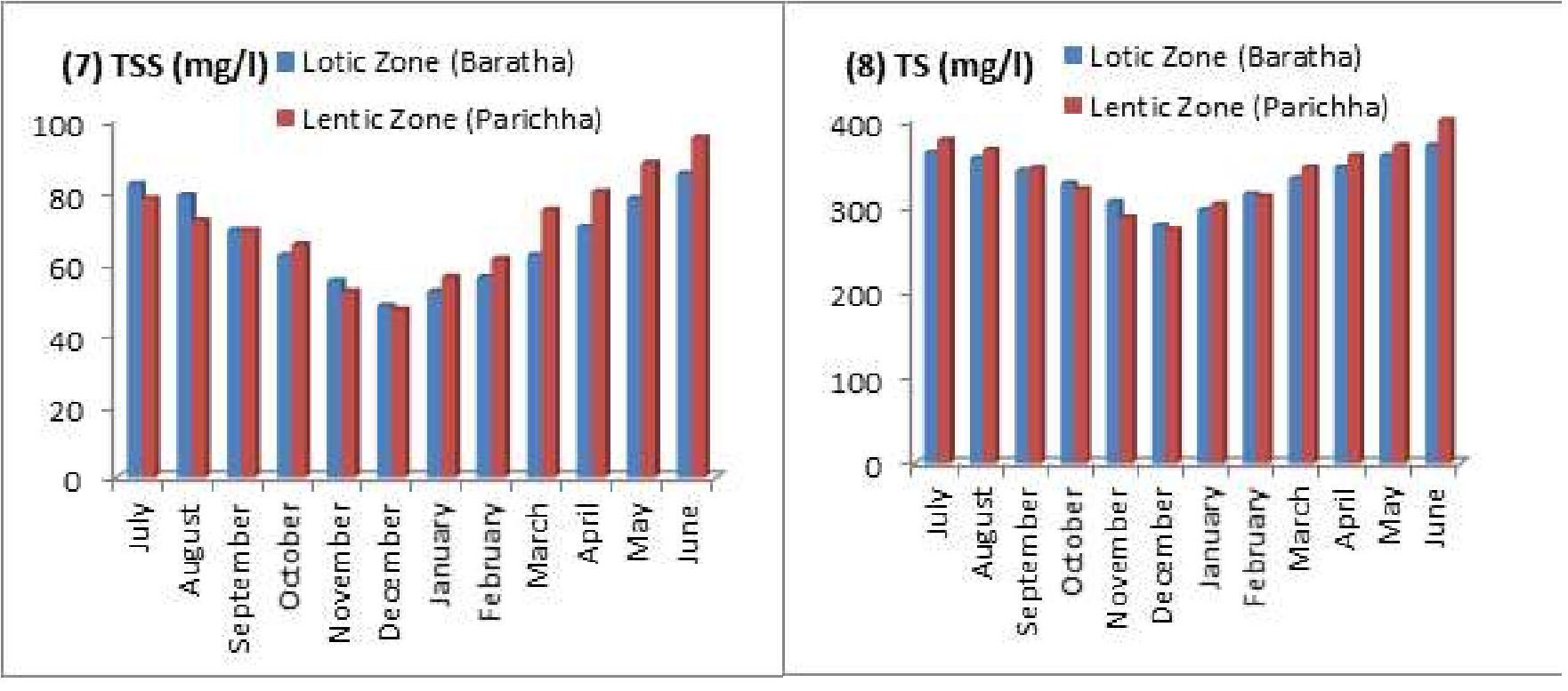

(9) DO (mg/l) Lotic Zone (Baratha)

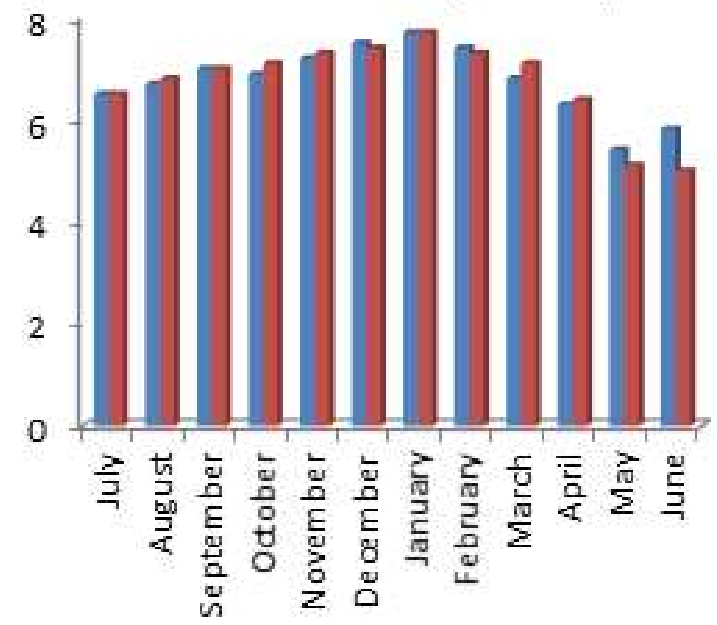

(10) TA (mg/l) = Lotic Zone (Baratha)

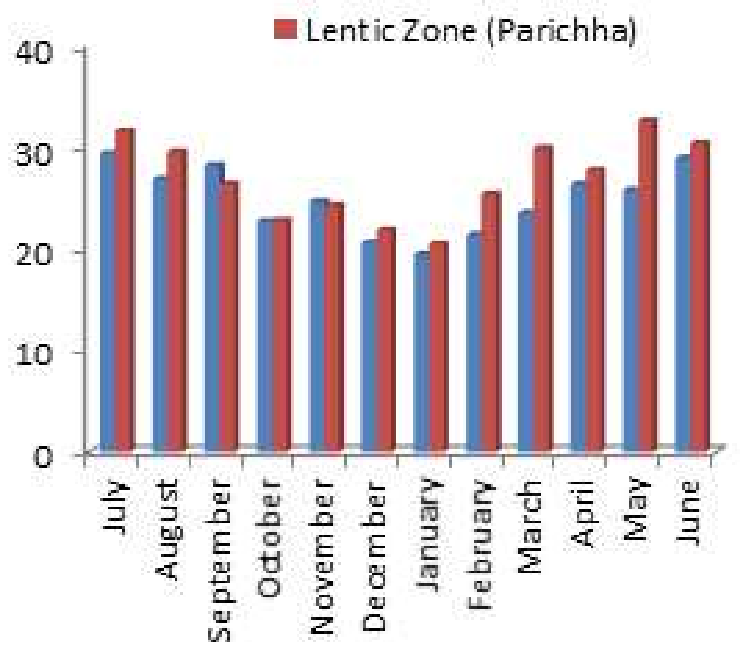

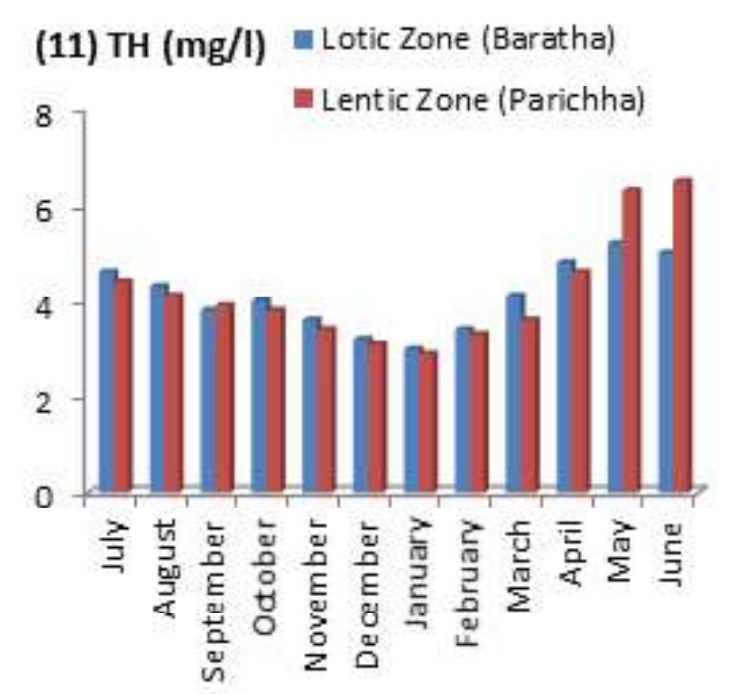

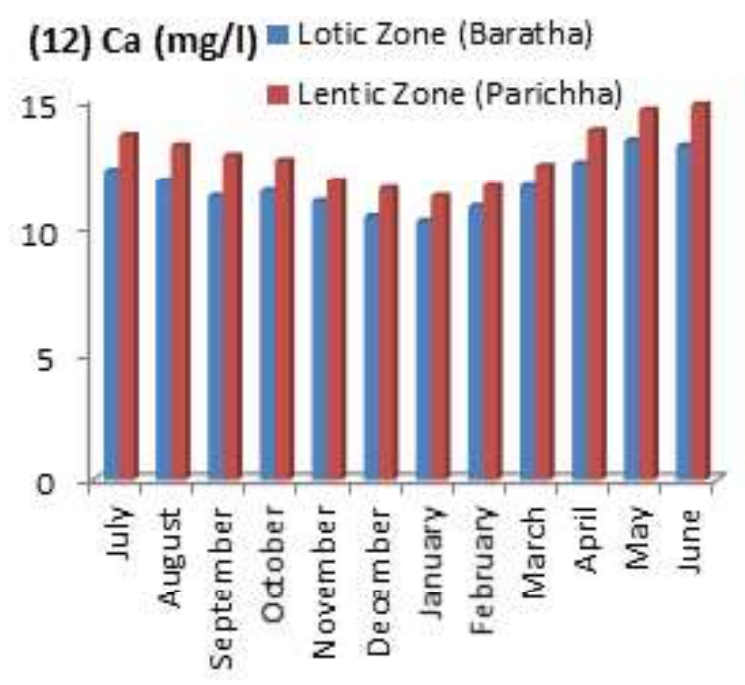

Figs : 7,8,9,10,11 and 12, comparative presentation of physico-chemical parameters of Lotic and Lentic Zones 

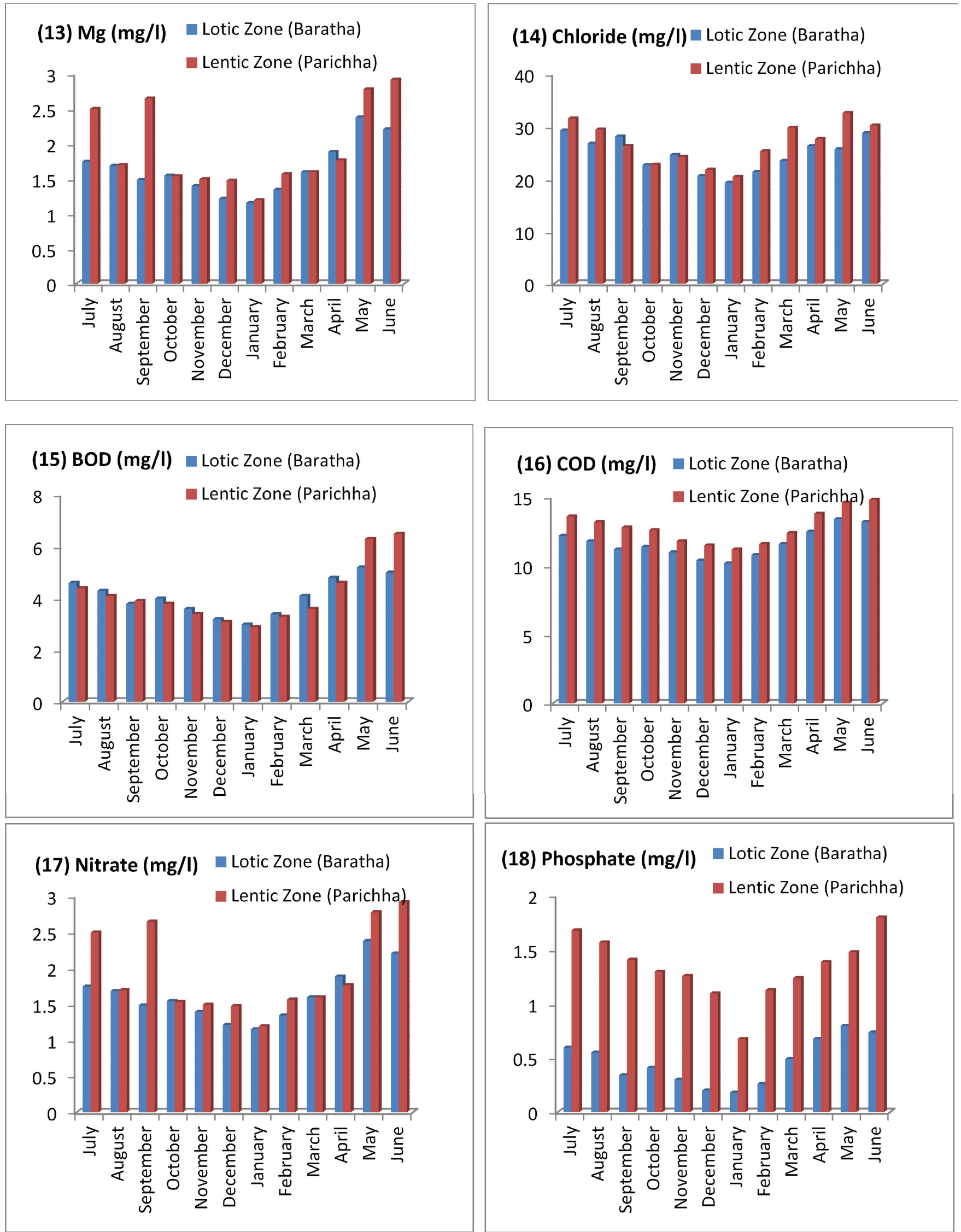

Figs : 13,14,15,16,17 and 18, comparative presentation of physico-chemical parameters of Lotic and Lentic Zones 
standard deviation range $(142.25 \pm 29.37)$. The total hardness range (Fig.11) of lotic zone was recorded maximum in May $(184 \mathrm{mg} / \mathrm{l})$ and minimum in December $(103 \mathrm{mg} / \mathrm{l})$ with their mean and standard deviation range (150.08 \pm 22.70$)$, while total hardness range of lentic zone was recorded maximum in June $(187 \mathrm{mg} / \mathrm{l})$ and minimum in December $(119 \mathrm{mg} / \mathrm{l})$ with their mean and standard deviation range (153.66 \pm 20.55$)$.

\section{$\mathrm{Ca}, \mathrm{Mg}$ and Chloride}

The calcium range (Fig.12) of lotic zone was recorded maximum in May (33.54 $\mathrm{mg} / \mathrm{l})$ and minimum in December $(26.20 \mathrm{mg} / \mathrm{l})$ with their mean and standard deviation range (29.04 \pm 2.32 ), while calcium range of lentic zone was recorded maximum in May $(36.83 \mathrm{mg} / \mathrm{l})$ and minimum in December $(21.60 \mathrm{mg} / \mathrm{l})$ with their mean and standard deviation range (29.84 \pm 5.38$)$. The magnesium range of lotic zone was recorded maximum in May (16.20 $\mathrm{mg} / \mathrm{l})$ and minimum in December $(10.44 \mathrm{mg} / \mathrm{l})$ with their mean and standard deviation range $(13.51 \pm 2.01)$, while magnesium range (Fig.13) of lentic zone was recorded maximum in June (16.74 mg/l) and minimum in December $(10.36 \mathrm{mg} / \mathrm{l})$ with their mean and standard deviation range (14.32 \pm 2.20 ). The chloride range (Fig.14) of lotic zone was recorded maximum in June $(28.80 \mathrm{mg} / \mathrm{l})$ and minimum in January $(19.34 \mathrm{mg} / \mathrm{l})$ with their mean and standard deviation range (24.77 \pm 3.29$)$, while chloride range of lentic zone was recorded maximum in May (32.62 mg/l) and minimum in January $(20.44 \mathrm{mg} / \mathrm{l})$ with their mean and standard deviation range $(26.85 \pm 3.99)$. Calcium, magnesium and chloride express their positively correlation with $\mathrm{EC}, \mathrm{pH}$ and temperature ${ }^{4}$.

\section{$B O D$ and COD}

BOD and COD parameters are considered as water pollution touchstone of any aquatic belt. The BOD range (Fig.15) of lotic zone was recorded maximum in May $(5.2 \mathrm{mg} / \mathrm{l})$ and minimum in January $(3.0 \mathrm{mg} / \mathrm{l})$ with their mean and standard deviation range $(4.08 \pm 0.71)$, while BOD range of lotic zone was recorded maximum in June $(6.5 \mathrm{mg} / \mathrm{l})$ and minimum in January $(2.9 \mathrm{mg} / \mathrm{l})$ with their mean and standard deviation range $(4.15 \pm 1.16)$. The COD range (Fig.16) of lotic zone was recorded maximum in May $(13.4 \mathrm{mg} / \mathrm{l})$ and minimum in January $(10.2 \mathrm{mg} / \mathrm{l})$ with their mean and standard deviation range $(11.64 \pm 1.02)$, while COD range of lentic zone was recorded maximum in June $(14.8 \mathrm{mg} / \mathrm{l})$ and minimum in January $(11.2 \mathrm{mg} / \mathrm{l})$ with their mean and standard deviation range (12.82 \pm 1.20$)$. Our findings are also accordance with several workers ${ }^{9,15}$.

\section{Nitrate and Phosphate}

The nitrate range (Fig.17) of lotic zone was recorded maximum in May $(2.38 \mathrm{mg} / \mathrm{l})$ and minimum in December $(1.22 \mathrm{mg} / \mathrm{l})$ with their mean and standard deviation range $(1.64 \pm 0.37)$, while nitrate range of lentic zone was recorded maximum in June $(2.92 \mathrm{mg} / \mathrm{l})$ and minimum in January $(1.20 \mathrm{mg} / \mathrm{l})$ with their mean and standard deviation range (1.93 \pm 0.59$)$. The phosphate range (Fig. 18) of lotic zone was recorded maximum in May $(0.80 \mathrm{mg} / \mathrm{l})$ and minimum in January $(0.18 \mathrm{mg} / \mathrm{l})$ with their mean and standard deviation range $(0.46 \pm 0.21)$, while phosphate range of lentic zone was recorded maximum in June (1.80 $\mathrm{mg} / \mathrm{l})$ and minimum in January $(0.68 \mathrm{mg} / \mathrm{l})$ with their mean and standard deviation range $(1.33 \pm 0.29)$.

\section{Conclusion}

Water of both zones i.e. lotic and lentic were found completely alkaline. Most of the water parameters like electrolytes, alkalinity, hardness, calcium, magnesium, chloride, BOD, COD and TDS were found higher in lentic zone than lotic zone due to stagnancy of natural flow of river water. The main cause of slightly polluted lentic zone (Parichha) is due to onthropogonic activities such as bathing in the river, washing clothes, household and agricultural waste runoff that were vigorously operated in the lotic zone (Baratha). Apart from this, the discharge effluent of the power plant situated at Parichha dam is also a valuable listed reason for the partial increased water pollution in the lentic zone. Hence in our research finding, lentic zone found to be slightly polluted compared to lotic zones of selected sampling station. However most of the ranges of water parameters found to be under the permissible limit hence both zones can be directly used for irrigation and fisheries sector but not for drinking purpose. To use it as drinking purpose, it must first be completely treated with standardized treatment, and only then can be used as drinkable water.

\section{References}

1. Adoni AD. Workbook on Limnology, Published by Pratibha Publication, Sagar, Madhya Pradesh. 1985; pp. 1-216.

2. Alam SK. Hydrobiological and physico-chemical analysis of the river Yamuna at Kalpi, district Jalaun, U.P. India. Ph.D. Thesis (Zoology), Bundelkhand University, Jhansi. 2013.

3. APHA. American Public Health Association, standards methods for the examination of water and waste water, Washington, D.C. 1998; 20 ed. 
4. Arya S, Devi A and Kumar VA. Comparative Study of Water quality Assessment of Parichha and Sukma Dukma Dams, Jhansi, India, International Journal of Advanced Engineering Technology. 2013; 4(4): 45-50.

5. Hussain G, Tharani M, Hussain A and Ahmad Y. Studies of Physico-chemical characteristics of river Betwa (M.P.), Indo American Journal of Pharmaceutical Sciences. 2017; 2(5):881-885.

6. Kulshrestha M, Singh B and Pani S. Impact of Industrial Effluents on water quality of Betwa river near Mandideep, Raisen, International Journal of Advanced Research in Biological Sciences. 2017; 4(4):169-181.

7. Kumar M, Singh R, Chaurasia S and Khare PK. Physico-chemical examination of Lotic water of River Yamuna at Kalpi, district Jalaun, Uttar Pradesh, India, Journal of Environmental Research and Development. 2016; 10(3):529536.

8. Mishra A, Tripathi S, Kumar V, Pandey HC and Sharma KK. Analysis of Physico-chemical properties of river water at Bundelkhand region of India, Agricultural Science Research Journal. 2016; 6(1):6-11.

9. Murhekar $\mathrm{GH}$. Determination of Physico-chemical parameters of Surface water samples in and around Akot city, International research Journal in Chemistry and Environment. 2011; 1(2):183-187.

10. Patel A and Datar M. Seasonal variation of Physico-chemical characterstics of River Betwa in Vidisha District, International Journal of Science, Environment. 2014; 3(6):2205-2214.

11. Perlman,H. Water Density, In the USGS Water Science School, Retrieved from http://water.usgs.gov/edu/ density.html. 2013.

12. Tripathi DM and Tripathi S. Ecological assessment of water quality of Betwa River in Jhansi District, Environmental Pollution and Protection. 2017; 2(1):1-5.

13. Trivedi RK and Goel PK. Chemistry and Biological methods for water pollution studies, Published by Environmental Publication, Kerad.1986;pp.1-246.

14. Verma S. Seasonal Variation of Water quality in Betwa River at Bundelkhand Region, India, Global Journal of Environmental Research. 2009; 3(3):164-168.

15. Vishwakarma S, Verma A and Saxena G. Assessment of water quality of Betwa river, Madhya Pradesh, India, International Journal of Water resources and Environmental Engineering. 2013; 5(4):217-222.

16. Zaidi $J$ and Pal A. Influence of temperature on Physico-chemical properties of fresh water ecosystem of Bundelkhand region of Uttar Pradesh, India, International Journal of Current Research in Chemistry and Pharmaceutical Sciences. 2015; 2(3):1-8.

17. Zaidi J, Ganesh S and Pal A. (2011) Assessment of water quality in river Betwa and Pahuj around Jhansi city, Uttar Pradesh, India, International Journal of Current Research. 2011; 3(5):027-031. 